\title{
RIP kinase 1-dependent endothelial necroptosis underlies systemic inflammatory response syndrome
}

\author{
Matija Zelic, ${ }^{1}$ Justine E. Roderick,, Joanne A. O'Donnell, ${ }^{1}$ Jesse Lehman, ${ }^{1}$ Sung Eun Lim,, ${ }^{1}$ Harish P. Janardhan, ${ }^{2}$ \\ Chinmay M. Trivedi, ${ }^{2}$ Manolis Pasparakis, ${ }^{3}$ and Michelle A. Kelliher ${ }^{1}$ \\ 'Department of Molecular, Cell and Cancer Biology, and 2Division of Cardiovascular Medicine and Department of Medicine, Program in Innate Immunity, University of Massachusetts Medical School, \\ Worcester, Massachusetts, USA. Institute for Cenetics, Centre for Molecular Medicine (CMMC), and Cologne Excellence Cluster on Cellular Stress Responses in Aging-Associated Diseases (CECAD), \\ University of Cologne, Cologne, Germany.
}

\begin{abstract}
Receptor interacting protein kinase 1 (RIPK1) has important kinase-dependent and kinase-independent scaffolding functions that activate or prevent apoptosis or necroptosis in a cell context-dependent manner. The kinase activity of RIPK1 mediates hypothermia and lethality in a mouse model of TNF-induced shock, reflecting the hyperinflammatory state of systemic inflammatory response syndrome (SIRS), where the proinflammatory "cytokine storm" has long been viewed as detrimental. Here, we demonstrate that cytokine and chemokine levels did not predict survival and, importantly, that kinase-inactive Ripk $1^{\text {D138N/D138N }}$ hematopoietic cells afforded little protection from TNF- or TNF/zVAD-induced shock in reconstituted mice. Unexpectedly, RIPK1 kinase-inactive mice transplanted with WT hematopoietic cells remained resistant to TNF-induced shock, revealing that a nonhematopoietic lineage mediated protection. TNF-treated Ripk $1^{0138 N / D 138 N}$ mice exhibited no significant increases in intestinal or vascular permeability, nor did they activate the clotting cascade. We show that TNF administration damaged the liver vascular endothelium and induced phosphorylated mixed lineage kinase domain-like (phospho-MLKL) reactivity in endothelial cells isolated from TNF/ZVAD-treated WT, but not Ripk1 ${ }^{0138 N / D 138 N}$, mice. These data reveal that the tissue damage present in this SIRS model is reflected, in part, by breaks in the vasculature due to endothelial cell necroptosis and thereby predict that RIPK1 kinase inhibitors may provide clinical benefit to shock and/or sepsis patients.
\end{abstract}

\section{Introduction}

The serine/threonine receptor interacting protein kinase 1 (RIPK1) plays a prominent role in proinflammatory and cell-survival signaling by mediating optimal NF- $\mathrm{KB}$ and MAPK pathway activation downstream of TNF and TRIF-dependent TLRs. In addition to prosurvival signaling, RIPK1 can induce caspase-8-dependent apoptosis or caspase-independent necroptosis. Under conditions of caspase inhibition, the related RIPK1 and RIPK3 kinases interact through their RIP homotypic interaction motif (RHIM), resulting in their auto- and trans-phosphorylation and RIPK3-mediated phosphorylation of the downstream pseudo-kinase mixed lineage kinase domain-like (MLKL). Phosphorylation of MLKL results in its oligomerization and translocation to the plasma membrane. Activated MLKL mediates membrane permeabilization by as-yet-unclear mechanisms, leading to the release of damageassociated molecular patterns (DAMPs) and necroptosis $(1,2)$. ATP, HMGB1, heat shock proteins, and other DAMPs are recognized by innate immune cells and can initiate or further amplify proinflammatory cytokine and chemokine production; thus, necroptosis is thought to be a more proinflammatory form of cell death compared with apoptosis (3). Necroptosis requires the kinase activities of RIPK1 and RIPK3 and can be activated down-

Conflict of interest: The authors have declared that no conflict of interest exists. Submitted: July 14, 2017; Accepted: March 6, 2018.

Reference information: / Clin Invest. 2018;128(5):2064-2075.

https://doi.org/10.1172/JCI96147. stream of death receptors (TNF, FAS, TRAIL), TLRs 3 and 4, type I or II interferon receptors (IFN- $\alpha \mathrm{R}$ or IFN- $\gamma \mathrm{R}$ ), and the cytosolic viral sensor Z-DNA-binding protein 1 (ZBP1) $(1,2)$.

While the kinase activity of RIPK1 was initially thought to only be required for necroptotic death, recent in vitro and in vivo genetic data have shown a context- and cell type-dependent role for RIPK1 and its kinase activity in activating or suppressing both apoptosis and necroptosis. Ripk1 $1^{-/-}$mice die at birth (4), but are rescued by codeletion of Casp 8 and Ripk3, demonstrating that RIPK1 suppresses caspase-8-dependent apoptosis and RIPK3dependent necroptosis during development (5-7). Furthermore, tissue-specific deletion of RIPK1 revealed that, in adult animals, RIPK1 primarily suppresses apoptosis in the intestine and necroptosis in the skin and hematopoietic system (6, 8-10). Necroptosis can proceed independently of RIPK1 in certain cell types, as RHIM-containing ZBP1 or TLR3/4 adapter TRIF can directly interact with RIPK3 (11-13). Since Ripk3-/- and $\mathrm{Mlkl}^{-/-}$mice are viable (14-16), studies using these mice have implicated necroptosis in various inflammatory, neurodegenerative, and infectious diseases (1, 2). RIPK1 kinase-dependent effects were initially interrogated using the small molecule inhibitor of RIPK1 kinase activity, Necrostatin-1 (Nec-1) (17, 18); however, interpreting these in vivo studies was limited by compound instability and off-target effects (19). The recent generation of kinase-inactive Ripk1 $1^{D 138 N / D 138 N}$ and Ripk1 $1^{K 45 A / K 45 A}$ mice has elucidated the role of RIPK1 kinase activity in necroptosis and in mouse models of acute and chronic inflam-

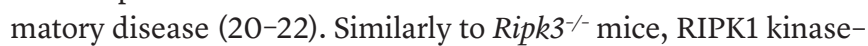



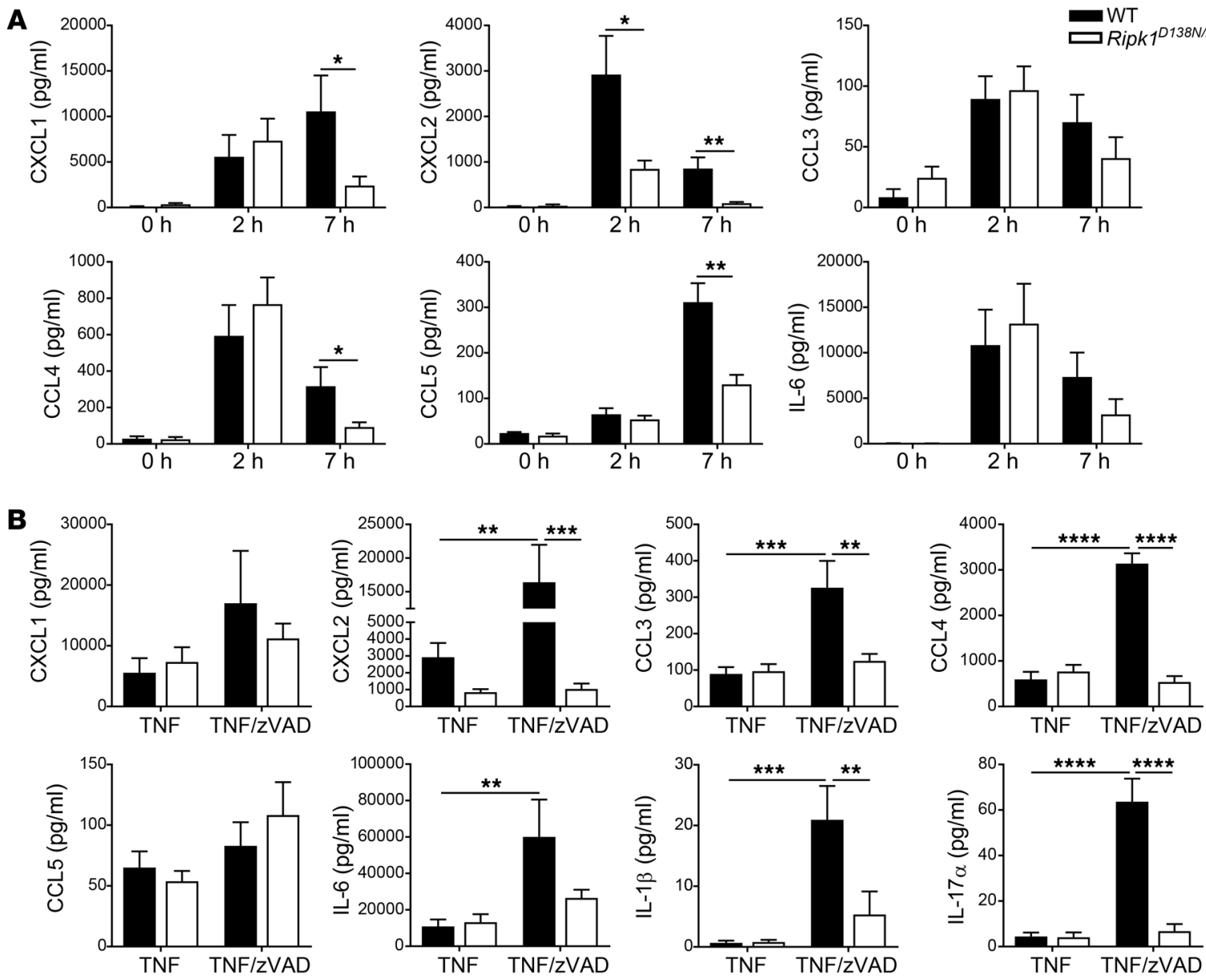

Figure 1. Ripk $1^{138 N / 0138 N}$ mice exhibit impaired cytokine and chemokine production in response to TNF and TNF/zVAD administration in vivo. (A) Plasma cytokine and chemokine levels in WT and Ripk $1^{138 N / D 138 N}$ mice 2 and 7 hours after i.v. TNF administration $(n=8-9)$ or (B) 2 hours after i.v. TNF or TNF/zVAD administration $(n=6-9)$. TNF-induced levels are repeated from $A$. Data are shown as mean \pm SEM. ${ }^{*} P<0.05 ;{ }^{* *} P<0.01 ;{ }^{* *} P<0.001 ;{ }^{* * * *} P<0.0001$, 2-tailed Student's $t$ test (A) or 2-way ANOVA with post hoc Tukey's test (B).

inactive $R i p k 1^{D 138 N / D 138 N}$ mice are protected from TNF- or TNF/ zVAD-induced acute shock; a model of systemic inflammatory response syndrome (SIRS) $(21,23)$.

SIRS can be triggered by sterile inflammation, including injury from burns, trauma, or acute pancreatitis, and upon infection, can lead to sepsis and septic shock $(24,25)$. It has long been hypothesized that the "cytokine storm" drives shock, with excess production of proinflammatory cytokines such as TNF- $\alpha$ and IL-1 $\beta$ triggering vascular dysfunction and organ injury (24). However, attempts over the last couple of decades to block TNF or IL-1R signaling in septic patients have largely failed to provide a survival benefit (26-28).

Although necroptosis has recently been implicated in the pathogenesis of TNF-induced shock and SIRS (20-23), the precise cell types undergoing necroptosis and the relative contribution of the cytokine storm remain unclear. Here, we demonstrate that proinflammatory cytokine/chemokine levels do not correlate with hypothermia or survival in this mouse SIRS model and that nonhematopoietic RIPK1 kinase-inactive cells mediate resistance to TNF- or TNF/zVAD-induced shock. Additionally, we show that WT liver endothelial cells undergo MLKL activation and cell death, indicating that endothelial cell necroptosis contributes to increased vascular permeability, coagulation, and organ injury observed in TNF/zVAD-treated WT, but not RIPK1 kinase-inactive, mice.

\section{Results}

RIPK1 kinase activity regulates cytokine/chemokine production and neutrophil infiltration in response to TNF and TNF/zVAD. TNF shock mimics SIRS, which is thought to be driven by the proinflammatory cytokine storm. To assess the role of RIPK1 kinase activity in the cytokine storm, we analyzed proinflammatory cytokine and chemokine levels in the plasma of TNF- or TNF/zVAD-treated mice. Similar increases in CXCL1, CCL3, CCL4, CCL5, and IL-6 levels were observed in Ripk1 $1^{D 138 N / D 138 N}$ and WT mice at early time points after TNF administration (Figure 1A). However, CXCL2 levels were consistently decreased in Ripk1 $1^{D 138 N / D 138 N}$ mice, suggesting that RIPK1 kinase activity may be required for optimal TNF-induced CXCL2 production in vivo (Figure 1A). Cytokine and chemokine levels were consistently reduced at 7 hours in RIPK1 kinase-inactive mice compared with TNF-treated WT mice (Figure 1A). The addition of the pan-caspase inhibitor zVAD sensitizes WT mice to TNF shock and decreases time to lethality 
A

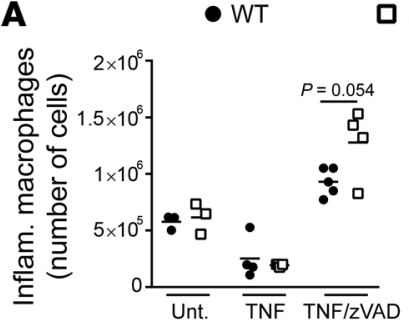

Ripk10138N/D138N

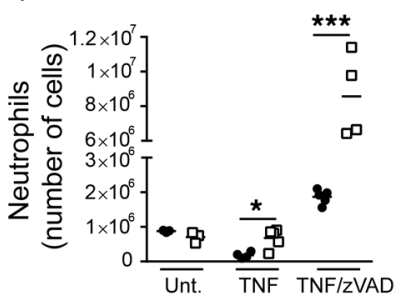

\section{C}
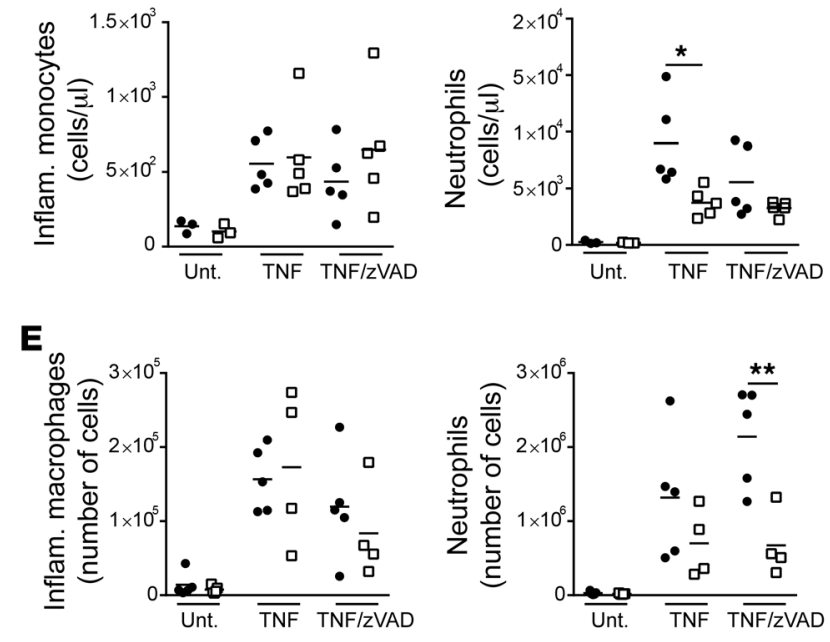

B
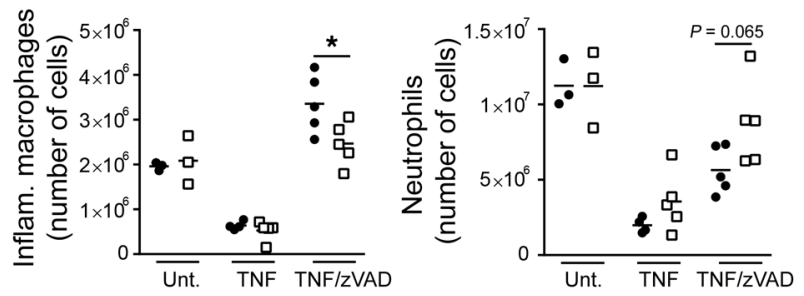

D
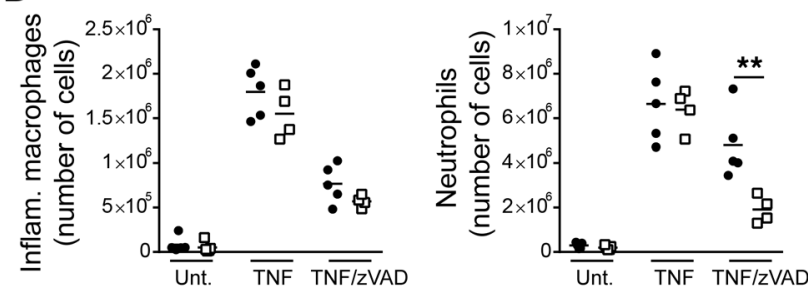

Figure 2. Impaired recruitment of Ripk $1^{0138 N / 138 N}$ inflammatory cells in response to TNF or TNF/zVAD administration. (A and B) Splenocytes and bone marrow cells were stained with antibodies, analyzed by flow cytometry, and the number of inflammatory (Inflam.) monocytes/macrophages or neutrophils determined. The number of cells for WT and Ripk $1^{138 N / 138 N}$ mice left untreated (Unt.) or treated with TNF (7 hours) or TNF/zVAD (4 hours) ( $\left.n=3-5\right)$ is shown. (C) The number of inflammatory monocytes or neutrophils in peripheral blood of WT and Ripk $1^{0138 N / 0138 N}$ mice left untreated or treated with TNF (7 hours) or TNF/zVAD (4 hours) is shown $(n=3-5)$. ( $\mathbf{D}$ and $\mathbf{E}$ ) The number of inflammatory macrophages or neutrophils in lung and liver of WT and Ripk $1^{1138 N / 0138 N}$ mice left untreated or treated with TNF (7 hours) or TNF/zVAD (4 hours) $(n=4-5)$ is shown. ${ }^{*} P<0.05 ;{ }^{*} P<0.01$; ${ }^{* * *} P<0.001,2$-tailed Student's t test.

$(21,23,29)$. To ascertain whether TNF/zVAD sensitization correlates with increased proinflammatory cytokine levels, we compared TNF- and TNF/zVAD-induced plasma cytokine/chemokine levels at 2 hours. Interestingly, WT mice treated with TNF/zVAD exhibited significantly elevated plasma cytokine and chemokine levels compared with those who received treatment with TNF alone, especially for CXCL2, CCL3, CCL4, and IL-6 (Figure 1B). IL-1 $\beta$ and IL-17 $\alpha$ levels, which are found to be increased in septic patients $(30,31)$, were elevated systemically in WT mice treated with TNF/zVAD, but not TNF (Figure 1B). While the addition of zVAD amplified cytokine and chemokine levels in WT mice, Ripk1 $1^{D 138 N / D 138 N}$ mice exhibited significantly lower plasma levels of proinflammatory cytokines and chemokines (Figure 1B). Overall, these results suggest that the plasma proinflammatory cytokine/ chemokine levels correlate with the kinetics of hypothermia and lethal shock. Furthermore, these data suggest that TNF/zVAD sensitizes mice to necroptotic death in vivo, which is postulated to be proinflammatory due to DAMP release (3).

Recently, the kinase activity of RIPK1 has been implicated in the transcriptional regulation of certain cytokines and chemokines in response to TNF/zVAD or LPS/zVAD treatment (22, 32-34). To test whether RIPK1 kinase is required for transcriptional activation of inflammatory cytokines/chemokines, we isolated primary bone marrow-derived macrophages (BMDMs) from WT and
Ripk1 $1^{1138 N / D 138 N}$ mice and stimulated them in vitro with TNF or TNF/ zVAD. We found that the kinase activity of RIPK1 was required for the induction and production of $C x c l 1, C x c l 2$, and $I l 6$, but not $C c l 5$, in response to TNF/zVAD (Supplemental Figure 1, A and B; supplemental material available online with this article; https://doi. org/10.1172/JCI96147DS1). Interestingly, Ripk1 $1^{D 138 N / D 138 N}$ BMDMs also exhibited reduced $\mathrm{Cxcl} 2$ expression and secretion when treated with TNF alone, indicating that RIPK1 kinase activity is necessary for optimal TNF-induced Cxcl2 upregulation in vitro (Supplemental Figure 1, A and B). Thus, RIPK1 kinase activity contributes to TNF- and TNF/zVAD-induced cytokine and chemokine transcription in primary BMDMs in vitro and may be responsible for reduced cytokine and chemokine levels during TNF shock in vivo.

To determine whether reductions in cytokine/chemokine levels in RIPK1 kinase-inactive mice result in decreased inflammatory cell mobilization and infiltration, we analyzed peripheral blood, bone marrow, spleen, lung, and liver for evidence of inflammatory macrophage and neutrophil recruitment in response to TNF or TNF/zVAD administration. Systemic TNF administration mobilized inflammatory macrophages and neutrophils from the spleens and bone marrow of WT and Ripk1 ${ }^{D 138 N / D 138 N}$ mice (Figure 2, A and B). However, more neutrophils were detected in Ripk1 ${ }^{D 138 N / D 138 N}$ spleen and bone marrow compared with those of TNF-treated WT mice (Figure 2, A and B). TNF-induced inflammatory monocyte 
A
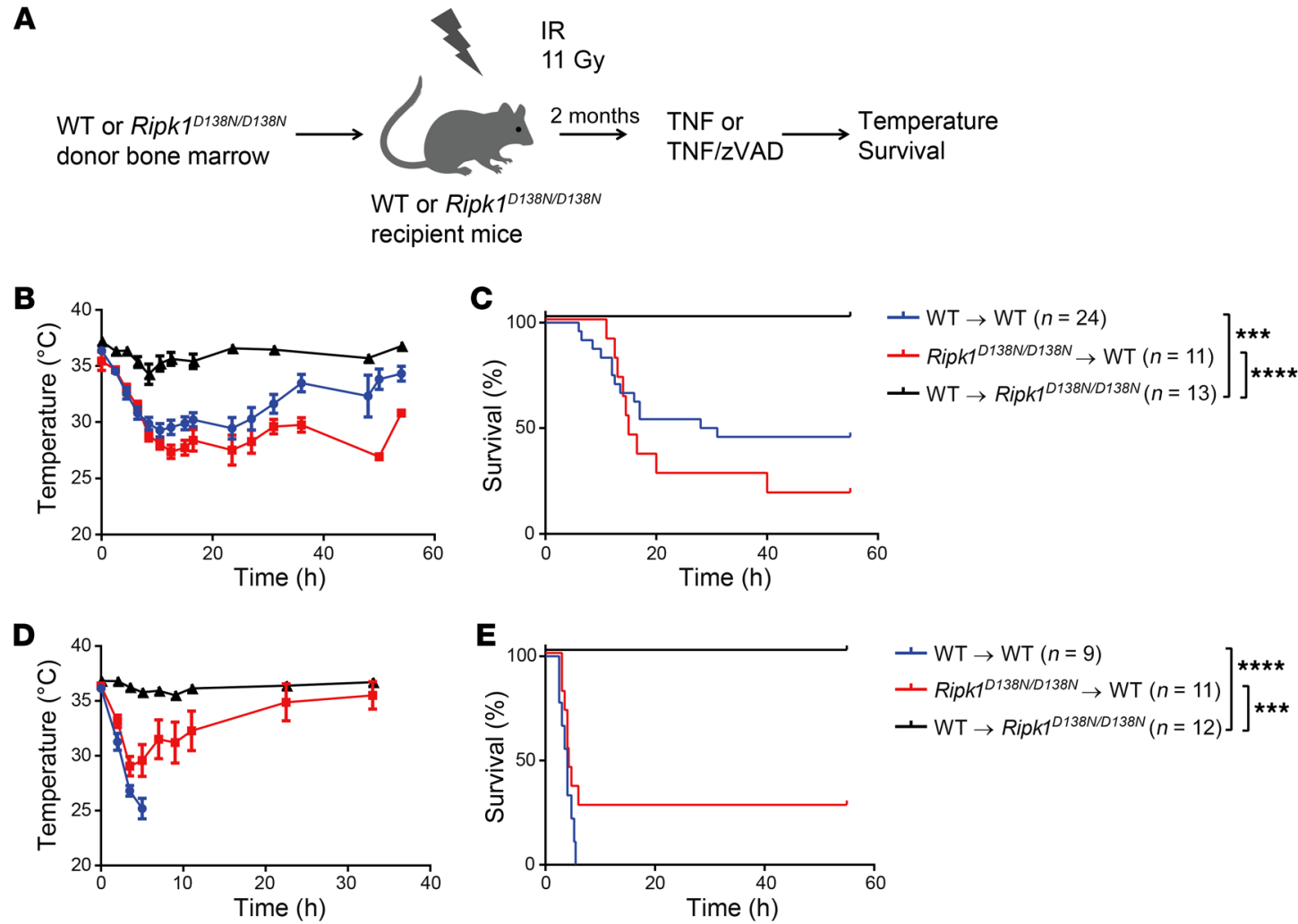

Figure 3. Nonhematopoietic RIPK1 kinase-inactive cells protect against TNF- and TNF/zVAD-induced shock. (A) Experimental design. IR, irradiation. Body temperature (B and $\mathbf{D}$ ) and Kaplan-Meier survival curves ( $\mathbf{C}$ and $\mathbf{E}$ ) after TNF- (B and $\mathbf{C}$ ) or TNF/zVAD-induced shock (D and E) are depicted for lethally irradiated WT and Ripk1 $1^{1138 N / 10138 N}$ mice reconstituted with WT or Ripk $1^{0138 N / 1138 N}$ bone marrow cells. Data are shown as mean \pm SEM. ${ }^{* * *} P<0.001$; ****P $<0.0001$, Mantel-Cox test.

recruitment to the blood and similar numbers of inflammatory monocytes/macrophages were detected in the lungs and liver of TNF-treated WT and Ripk1 ${ }^{D 138 N / D 138 N}$ mice (Figure 2, C-E). Consistent with reduced levels of the neutrophil chemoattractant CXCL2 (Figure 1A), TNF-treated Ripk1 $1^{138 N / D 138 N}$ mice exhibited decreased neutrophil mobilization from the bone marrow and spleen into the blood. However, inflammatory cell infiltration in lung and liver was similar for WT and Ripk1 $1^{\text {D188N/D138N }}$ mice treated with TNF (Figure 2). Although TNF/zVAD administration to WT mice resulted in a more robust cytokine/chemokine response (Figure 1B), this did not result in significant increases in inflammatory cell mobilization into blood, lung, or liver compared with treatment with TNF only (Figure 2, C-E). Although TNF/zVAD treatment significantly increased neutrophil numbers in the spleen and bone marrow, Ripk1 $1^{D 138 N / D 138 N}$ mice exhibited reduced neutrophil infiltration into lung and liver (Figure 2). Activated neutrophils release proteases and reactive oxygen species, thereby damaging organs and further increasing cytokine production (35). Thus, the reduced cytokine/ chemokine levels and impaired neutrophil mobilization observed in TNF- and TNF/zVAD-treated Ripk1 $1^{138 N / D 138 N}$ mice may be responsible for the protection.

RIPK1 kinase activity in a nonhematopoietic lineage is responsible for TNF- and TNF/zVAD-induced lethality. These data suggest that inflammatory cells mediate organ damage and predict that RIPK1 kinase-inactive hematopoietic cells provide protection in this SIRS model. To test this, we generated reciprocal bone marrow chimeras by transplanting WT or Ripk1 $1^{138 N / D 138 N}$ bone marrow cells into lethally irradiated syngeneic recipients (Figure 3A). Two months after reconstitution, RIPK1 kinase-inactive donor-derived cells repopulated irradiated recipients as effectively as WT cells (Supplemental Figure 2, A-C), indicating that RIPK1 kinase activity does not contribute to the cell-intrinsic survival function of RIPK1 in hematopoietic cells $(6,10)$. We next tested the TNF response, and as expected, WT controls developed hypothermia in response to TNF (Figure 3B) or TNF/zVAD (Figure 3D) administration and succumbed to shock. Unexpectedly, Ripk1 ${ }^{D 138 N / D 138 N} \rightarrow$ WT mice succumbed with kinetics similar to those of WT $\rightarrow$ WT controls in response to TNF (Figure 3C) or TNF/zVAD (Figure 3E) administration. Interestingly, $\mathrm{WT} \rightarrow$ Ripk1 $1^{D 138 N / D 138 \mathrm{~N}}$ mice were completely protected from TNF- or TNF/zVAD-induced shock (Figure 3, B-E). These data reveal that a nonhematopoietic Ripk1 ${ }^{138 N / D 138 N}$ lineage or lineages mediate protection from TNF- and TNF/zVAD-induced shock.

Cytokine levels and neutrophil infiltration do not correlate with survival. To further examine any contribution of the cytokine storm, we measured proinflammatory cytokine and chemokine levels in the plasma of TNF-treated reconstituted mice. In a manner comparable to what we observed in nonreconstituted mice, TNF treatment similarly upregulated cytokine and chemokine levels at 2 hours in all the bone marrow chimeras, notably revealing that RIPK1 kinase activity does not appear to regulate cytokine transcription during TNF shock (Figure 4A). While cytokine/chemokine levels were further elevated in WT controls 7 hours after TNF administration, 
$\mathbf{A}$
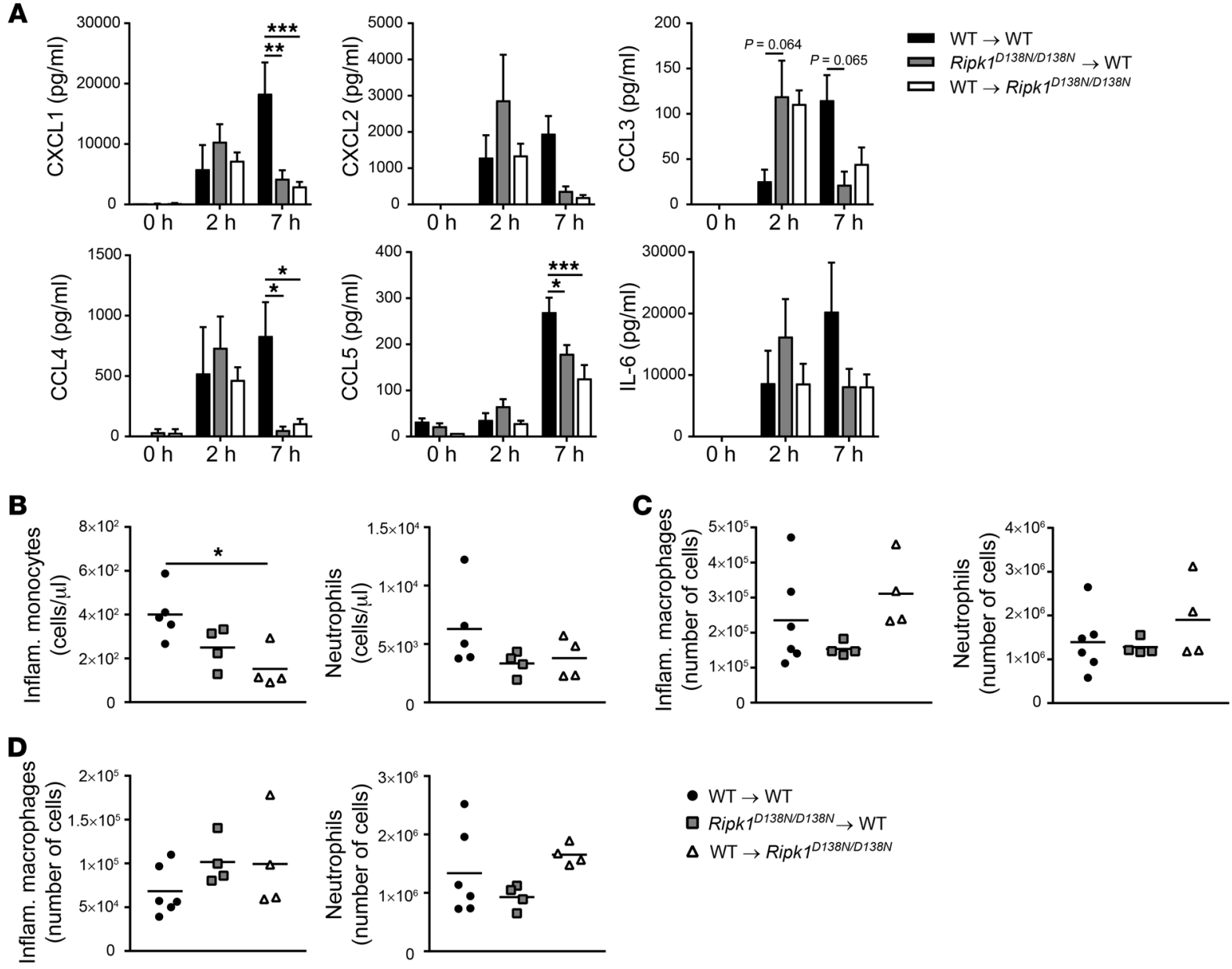

Figure 4. Proinflammatory cytokines and inflammatory cell recruitment do not correlate with survival in a TNF-induced model of shock. (A) Plasma cytokine and chemokine levels in reconstituted mice 2 and 7 hours after i.v. TNF administration $(n=4-7)$. Graphs depicting the number of inflammatory

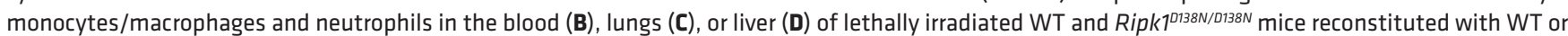
Ripk $1{ }^{138 N / 1138 N}$ bone marrow cells as measured by flow cytometry 8 hours after systemic TNF administration $(n=4-5)$. Data are shown as mean \pm SEM. ${ }^{*} P<0.05 ;{ }^{* *} P<0.01 ;{ }^{* *} P<0.001,2$-way (A) or 1-way ANOVA with post hoc Tukey's test (B-D).

unexpectedly, both sensitive Ripk1 $1^{D 138 N / D 138 N} \rightarrow \mathrm{WT}$ and resistant $\mathrm{WT} \rightarrow R i p k 1^{138 N / D 138 N}$ chimeras exhibited significant decreases in cytokine/chemokine levels (Figure 4A), demonstrating that cytokine/chemokine levels do not predict survival. These data also suggest that RIPK1 kinase-inactive hosts lack a secondary trigger, potentially provided by the necroptosis of nonhematopoietic cells, that may amplify proinflammatory signaling by feeding back on necroptosis-competent WT hematopoietic cells. Additionally, the data suggest that, in sensitive Ripk1 $1^{D 138 N / D 138 N} \rightarrow$ WT mice, DAMPs released from necroptotic WT nonhematopoietic cells fail to amplify cytokine/chemokine levels to levels observed in WT $\rightarrow \mathrm{WT}$ mice, potentially because Ripk1 $1^{D 138 N / D 138 N}$ hematopoietic cells do not undergo necroptosis (Figure 4A). Furthermore, we found that TNF/zVAD treatment resulted in greater increases in cytokine/chemokine levels at 2 hours in both sensitive Ripk1 ${ }^{D 138 N / D 138 N}$ $\rightarrow$ WT mice and resistant WT $\rightarrow$ Ripk1 $1^{D 138 N / D 138 N}$ chimeras compared with treatment with TNF only (Supplemental Figure 3), suggesting that necroptosis amplifies proinflammatory cytokine/chemokine production. Additionally, maximal cytokine/chemokine production requires RIPK1 kinase activity in both hematopoietic and non- hematopoietic lineages, whereas a lack of RIPK1 kinase activity in a nonhematopoietic lineage provides protection.

We observed similar mobilization and infiltration of inflammatory monocytes/macrophages and neutrophils into the blood, lungs, and liver of all TNF-treated reconstituted groups (Figure 4, B-D). In fact, more inflammatory macrophages and neutrophils infiltrated the lung and liver, respectively, of protected WT $\rightarrow$ Ripk1 $1^{D 138 N / D 138 N}$ chimeras compared with sensitive Ripk1 $1^{D 138 N / D 138 N}$ $\rightarrow$ WT mice. These data suggest that a lack of RIPK1 kinase activity in either hematopoietic or nonhematopoietic cells does not abrogate TNF-induced inflammatory cell recruitment.

TNF-induced increases in intestinal permeability require RIPK1 kinase activity. Since RIPK1 kinase-inactive hematopoietic cells proved not to be the critical lineage mediating protection from TNF shock (Figure 3), we examined other lineages affected by TNF administration. TNF receptor 1 (TNFR1) expression levels in intestinal epithelial cells have been shown to correlate with TNFinduced increases in intestinal permeability and overall lethality in mice $(36,37)$. To assess intestinal permeability, we measured the plasma fluorescence levels of orally gavaged FITC-conjugated 
A

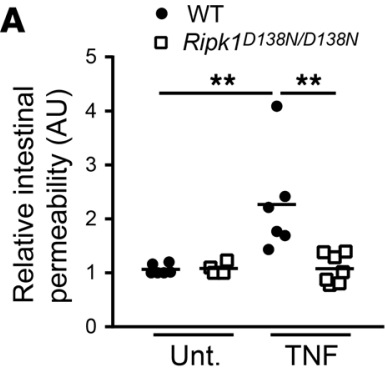

C
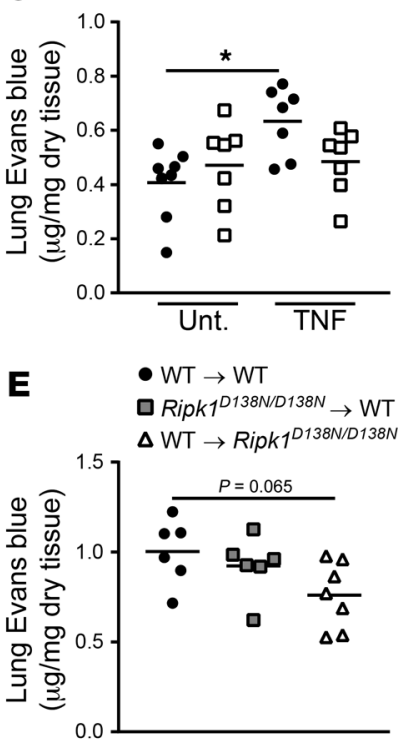

B
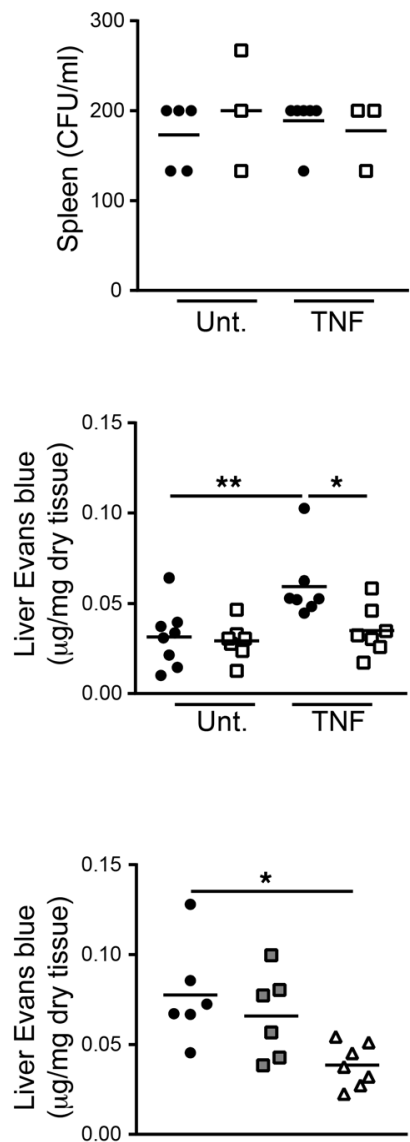
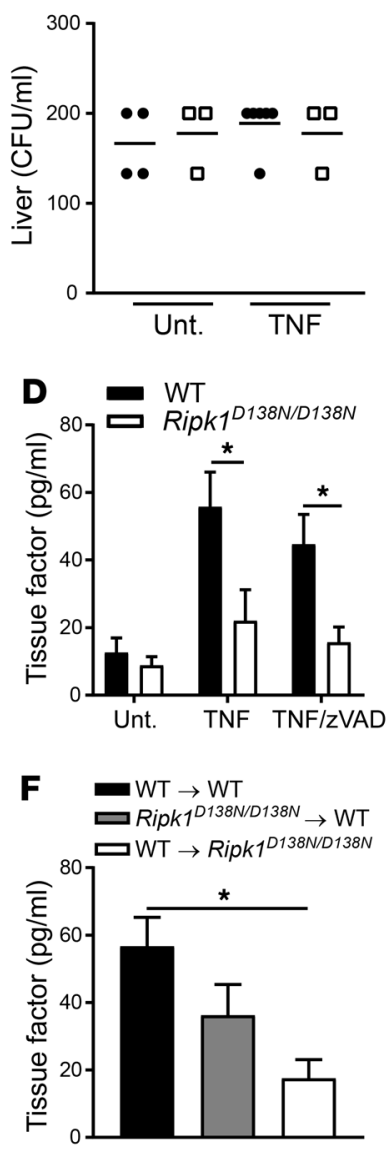

Figure 5. Nonhematopoietic RIPK1 kinase-inactive cells protect against TNF-induced intestinal and vascular hyperpermeability and coagulation. (A) FITC-labeled dextran beads were administered to WT and Ripk10138N/D138N mice by oral gavage 2 hours after TNF injection. Blood was isolated by cardiac puncture and the levels of FITC-dextran in plasma measured by fluorometry $(n=4-7)$. (B) Bacterial growth in spleen and liver lysates isolated from untreated WT and Ripk1 $1^{0138 N / 0138 N}$ mice or 10 hours after injection with $15 \mu \mathrm{g}$ TNF $(n=3-6)$. (C) WT and Ripk $7^{1138 N / D 138 N}$ mice were injected with TNF and Evans blue dye, organs were collected, and the amount of dye extracted from the lung and liver was measured by spectrophotometry ( $n=7-8)$. (D) Tissue factor levels were determined by ELISA in the plasma of TNFor TNF/zVAD-treated WT and Ripk17138N/D138N mice $(n=3-7)$. (E) WT and Ripk10138N/D138N reconstituted mice were injected with TNF and Evans blue dye, and the amount of dye extracted from the lung and liver was measured by spectrophotometry $(n=6-7)$. (F) Tissue factor levels in the plasma of reconstituted WT and Ripk10138N/D138N mice treated with TNF $(n=3-5)$. Data are shown as mean \pm SEM. ${ }^{*} P<0.05$; ${ }^{*} P<0.01$, 2-way ANOVA with post hoc Tukey's test (A and C), 2-tailed Student's $t$ test (D), or 1-way ANOVA with post hoc Tukey's test ( $E$ and $F$ ). dextran beads following TNF administration. WT mice exhibited a significant increase in intestinal permeability, but no increase in intestinal permeability was observed in Ripk1 $1^{D 138 N / D 138 N}$ mice upon TNF administration (Figure 5A). Although Ripk1 $1^{D 138 N / D 138 N}$ mice were protected from TNF-induced lethality, cleaved caspase-3 staining revealed similar numbers of apoptotic colonocytes in WT and RIPK1 kinase-inactive mice (Supplemental Figure 4B). Interestingly, WT mice had slightly elevated numbers of cleaved caspase-3-positive cells in the ileum, suggesting that some intestinal epithelial cells may undergo RIPK1 kinase-mediated apoptosis in response to TNF (Supplemental Figure 4A). Importantly, $\mathrm{TNF} / \mathrm{zVAD}$ administration similarly suppressed ileal and colonic apoptosis in WT and Ripk1 $1^{D 138 N / D 138 N}$ mice, suggesting that intestinal apoptosis is not responsible for the lethality observed in WT mice (Supplemental Figure 4, A and B). Since intestinal leakage could disseminate the gut microflora systemically, activate TLR signaling, and amplify inflammatory cytokine production (37), we quantified bacterial growth in spleen and liver lysates from untreated and TNF-treated WT and Ripk1 $1^{D 138 N / D 138 N}$ mice (Figure 5B). Although TNF augmented intestinal permeability, we could not detect an increase in bacterial colonies from spleen and liver lysates when WT mice were treated with TNF (Figure 5B), indicating that changes in intestinal permeability were not sufficient to induce systemic bacteremia in this i.v. TNF shock model.

RIPK1 kinase activity is required for TNF-induced increases in vascular permeability and clotting. In addition to increasing intes- tinal epithelial permeability, TNF stimulates vascular permeability and endothelial activation by upregulating the expression of adhesion molecules and by stimulating cytokine and chemokine production $(38,39)$. To quantitatively assess TNF-induced vascular permeability changes, we measured the extravasation of Evans blue dye. TNF administration induced significant increases in vascular permeability, resulting in Evans blue dye leakage into the lungs and liver of WT mice, while Ripk1 $1^{\text {D138N/D138N }}$ mice exhibited no detectable increase in vascular permeability (Figure 5C). Additionally, WT mice exhibited activation of the extrinsic coagulation cascade, as evidenced by significant increases in plasma tissue factor levels following TNF or TNF/zVAD administration (Figure 5D). To determine whether RIPK1 kinase activity in endothelial cells was responsible for the increased vascular permeability, we examined these vascular parameters in chimeric mice. WT $\rightarrow$ WT and Ripk1 ${ }^{D 138 N / D 138 N} \rightarrow$ WT chimeras, which remain sensitive to TNF shock, displayed increased vascular permeability in their lungs and liver (Figure 5E) and exhibited elevated tissue factor levels during TNF-induced shock (Figure 5F). Notably, WT $\rightarrow$ Ripk1 $1^{\text {D138N/D138N }}$ mice, which were protected from TNF-induced shock, showed no significant increase in TNF-induced vascular permeability and maintained basal levels of plasma tissue factor in response to TNF (Figure 5, E and F). These data suggest that RIPK1 kinase activity in endothelial cells is responsible for TNF-induced increases in vascular permeability and coagulation. Consistent with these data, histological examination of the livers in TNF- and TNF/ 
A
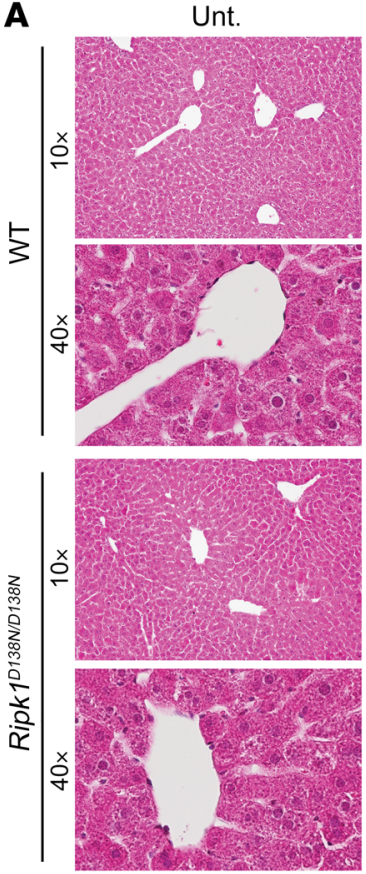

D

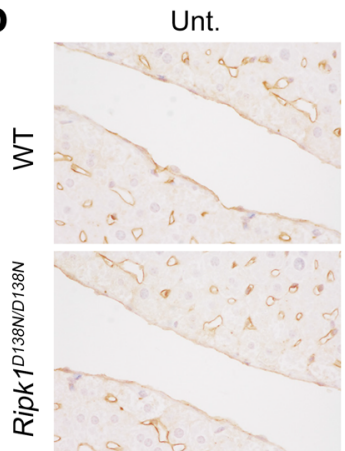

TNF
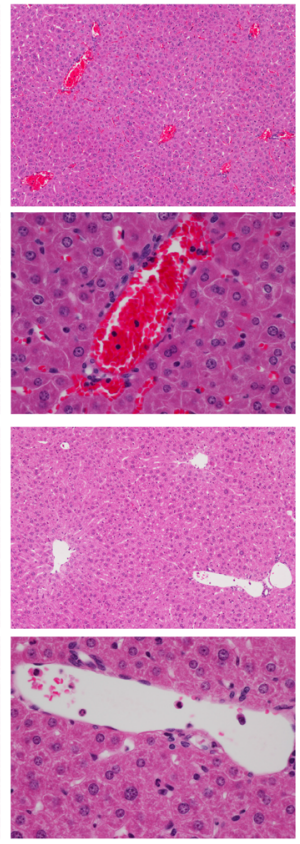

TNF

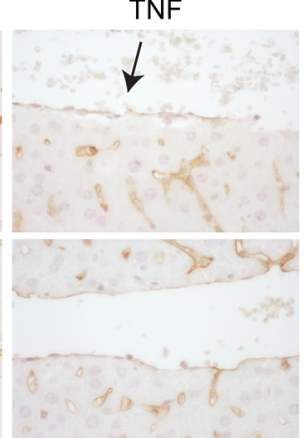

TNF/zVAD
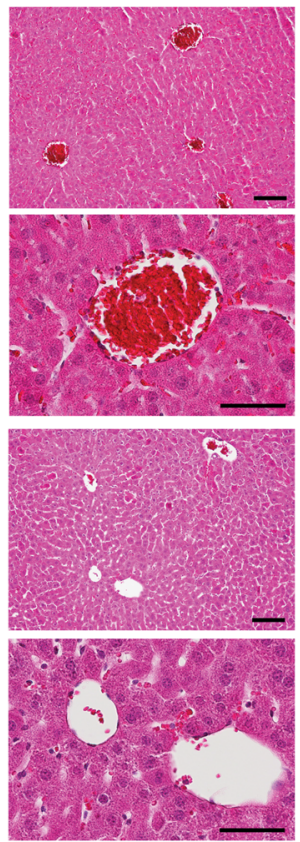

TNF/zVAD

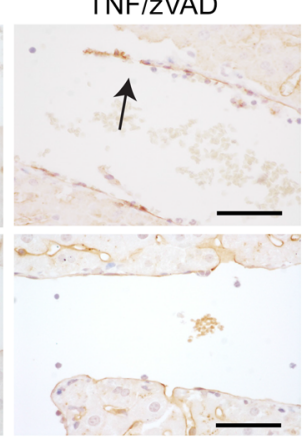

B

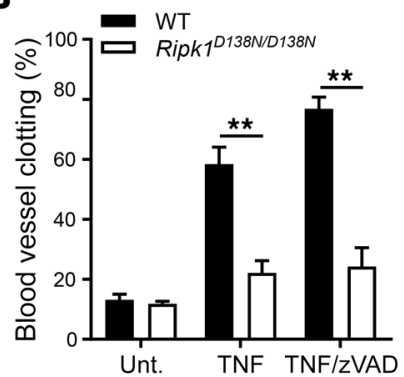

C

- WT

口 Ripk1 D138N/D138N

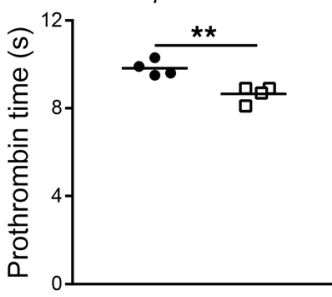

E

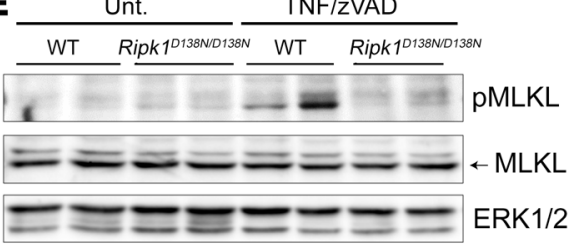

Figure 6. RIPK1 kinase-inactive mice are protected from TNF- or TNF/zVAD-induced coagulation and breaks in the liver endothelium, and from TNF/ zVAD-induced liver endothelial cell necrosis. (A) Representative images of H\&E-stained liver sections from WT or Ripk $1^{0138 N / D 138 N}$ mice left untreated or injected i.v. with TNF or TNF/zVAD ( $n=3-4$ mice). Scale bars: $100 \mu \mathrm{m}(\times 10) ; 50 \mu \mathrm{m}(\times 40)$. (B) Quantification of clotting observed in liver blood vessels of mice shown in A. (C) Citrated plasma was isolated from WT and Ripk $1^{1338 N / 138 N}$ mice 7 hours after TNF administration, and clot formation time was measured by the prothrombin time test $(n=4$ mice). (D) Representative images of endomucin-stained liver sections from untreated or TNF- or TNF/ zVAD-treated WT and Ripk10138N/D138N mice ( $n=3-4$ mice). Scale bars: $50 \mu \mathrm{m}(\times 40)$. (E) WT and Ripk10138N/D138N liver endothelial cells were isolated from untreated or TNF/zVAD-treated mice. A representative Western blot showing protein lysates probed with antibodies to phospho-MLKL, MLKL, and ERK1/2 is depicted. Lysates were generated and analyzed from 14 WT and 8 Ripk10138N/D138N TNF/zVAD-treated mice. Data are shown as mean \pm SEM. ${ }^{* *} P<0.01$, 2-tailed Student's $t$ test.

zVAD-treated WT mice revealed significant increases in blood vessel clotting compared with that in untreated controls (Figure 6, A and B). We observed neutrophil and erythrocyte adhesion to the endothelium and leakage of erythrocytes and leukocytes into the extravascular space due to increased vascular permeability (Figure 6A). Although we observed some evidence of neutrophil/erythrocyte adhesion and clotting in the TNF- and TNF/ zVAD-treated Ripk1 $1^{D 138 N / D 138 N}$ liver sections, there was a significant reduction in clotting in the liver blood vessels (Figure 6, A and B). To quantify clotting function, we performed a prothrombin time test. Blood isolated from TNF-treated WT mice took significantly longer to clot compared with that of RIPK1 kinase-inactive mice (Figure 6C), suggesting that coagulation factors were consumed in TNF-treated WT mice due to activation of the extrinsic clotting cascade. These data demonstrate that lack of RIPK1 kinase activity in endothelial cells protects from TNF- and TNF/zVAD-induced increases in vascular permeability and coagulation.

TNF-induced adhesion molecule expression does not require RIPK1 kinase activity. We hypothesized that RIPK1 kinase activity may regulate vascular permeability by directly mediating endothelial cell activation. To test this possibility, we isolated primary endothelial cells from the lungs of WT and Ripk1 $1388 N / D 138 N$ mice and measured Icam1, Vcam1, Sele, and Selp mRNA levels via realtime quantitative PCR. We detected no significant differences in TNF-induced Icam1, Vcam1, Sele, and Selp upregulation in WT and Ripk1 $1^{D 138 N / D 138 N}$ primary lung endothelial cells, indicating that 
RIPK1 kinase activity is not required for TNF-induced endothelial activation in vitro (Supplemental Figure 5A). Endothelial cells upregulate neutrophil chemokines CXCL1 and CXCL2 in response to TNF stimulation (39). Thus, we examined CXCL1 and CXCL2 expression and secretion, but failed to detect any significant differences in endothelial chemokine production between WT and RIPK1 kinase-inactive endothelial cells (Supplemental Figure 5, $\mathrm{A}$ and $\mathrm{B}$ ). We then assessed whether RIPK1 kinase activity influenced endothelial cell activation in vivo. After TNF or TNF/zVAD administration, we isolated RNA from WT and Ripk1 $1^{D 138 N / D 138 N}$ lung or liver and examined adhesion molecule and chemokine expression levels. We detected significant reductions in Sele, Selp, and Cxcl2 expression in TNF- or TNF/zVAD-treated Ripk1 $1^{\text {D138N/D138N }}$ mice; however, no consistent differences were observed in Icam1 or Vcam1 expression (Supplemental Figure 6, A and B). Although chemokine differences were less pronounced in TNF-treated livers, $\mathrm{TNF} / \mathrm{zVAD}$ treatment resulted in significant increases in $\mathrm{Cxcl} 2$ expression in WT compared with Ripk1 $1^{\text {D138N/D138N }}$ liver (Supplemental Figure 6B), perhaps reflective of increased plasma IL-1 $\beta$ and IL-17 $\alpha$ levels (40-42). While decreased chemokine mRNA levels in Ripk1 ${ }^{D 138 N / D 138 N}$ lung and liver correlated with reduced neutrophil infiltration during TNF- and TNF/zVAD-induced shock (Figure 2, $\mathrm{D}$ and $\mathrm{E}$ ), TNF- or TNF/zVAD-induced adhesion molecule upregulation in vivo did not appear to require RIPK1 kinase activity.

RIPK1 kinase activity mediates TNF/zVAD-induced liver endothelial cell necroptosis. Since the kinase activity of RIPK1 was not required for endothelial cell activation, we hypothesized that RIPK1 kinase-regulated endothelial cell death may be responsible for the increase in vascular permeability, coagulation, and organ damage observed in WT mice. To test whether endothelial cells undergo necroptotic death, we isolated primary lung endothelial cells from WT and Ripk1 $1^{D 138 N / D 138 N}$ mice and treated them with concentrations of TNF, Smac mimetic, and zVAD known to induce necroptosis in murine embryonic fibroblasts (43). Primary lung endothelial cells isolated from WT mice did not undergo TNF/Smac-induced apoptosis or TNF/zVAD-induced necroptosis, but were susceptible to necroptosis induced by TNF/Smac/zVAD treatment (Supplemental Figure 5C). In contrast, Ripk1 $1^{D 138 N / D 138 N}$ endothelial cells were resistant to TNF/Smac/zVAD-induced necroptosis (Supplemental Figure 5C). Collectively, these data demonstrate that RIPK1 kinase activity is required for TNF-induced endothelial necroptosis in vitro (Supplemental Figure 5C) and for increased vascular permeability in vivo (Figure 5), implicating RIPK1 kinase-dependent endothelial cell necroptosis in SIRS.

To examine this possibility, we stained liver sections with endomucin, an endothelial cell marker (44), after TNF or TNF/ zVAD administration (Figure 6D). In treated WT mice, we detected breaks in the endothelial barrier, indicating endothelial cell loss and/or detachment and increased permeability and extravasation of leukocytes into the interstitial space (Figure 6D). TNF/zVAD treatment induced endothelial detachment with evidence of leukocyte adherence and translocation in all 3 WT mice examined, whereas these morphological changes were observed in 2 of 4 WT mice treated with TNF. These histopathologic findings were not

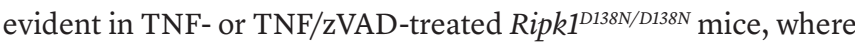
liver endothelial barrier integrity was maintained similarly to that in untreated mice (Figure 6D).
Due to a lack of reliable measures of necroptotic death in mice, we isolated primary liver endothelial cells from TNF- and TNF/ zVAD-treated WT and Ripk1 $1^{D 138 N / D 138 N}$ mice (80\% average purity, Supplemental Figure 7, A and B) and assessed MLKL activation. We consistently detected phospho-MLKL reactivity in endothelial cells isolated from TNF/zVAD-treated WT mice, but not in endothelial cells isolated from Ripk1 $1^{D 138 N / D 138 N}$ mice (Figure 6E). We were unable to detect phospho-MLKL reactivity in endothelial cells isolated from TNF-treated mice, where endothelial breaks were observed only in half the mice examined (Figure 6D). Although unclear in the TNF shock model, TNF/zVAD-induced shock is mediated by RIPK1-dependent liver endothelial cell necroptosis.

\section{Discussion}

Here we demonstrate that the resistance of RIPK1 kinase-inactive mice to TNF- and TNF/zVAD-induced shock is not due to hematopoietic cells and/or cytokine storm, but rather due to lack of endothelial cell necroptosis, which prevents breaks in the vascular endothelium, activation of the clotting cascade, and organ damage. Our results provide genetic evidence that RIPK1 kinase activity mediates endothelial cell necroptosis during TNF/zVAD-induced shock.

Cytokines such as IL-1 $\beta$ and IL-17 $\alpha$, and both apoptotic and necroptotic cell death, have been implicated in TNF-induced lethality $(21,23,45-47)$. As reported previously in Nec-1-treated WT mice and Ripk3 ${ }^{-/-}$mice (23), we found cytokine/chemokine induction to be normal in TNF-treated Ripk1 $1^{D 138 N / D 138 N}$ mice; however, these mice failed to sustain cytokine levels in response to TNF administration. Interestingly, we show that TNF/zVAD-induced shock augmented the proinflammatory response in WT, but not $R i p k 1^{D 138 N / D 138 N}$, mice (Figure 1B), supporting the notion that necroptosis amplifies proinflammatory signaling and exacerbates organ injury. Multiple studies have shown that RIPK3 deficiency or pharmacologic inhibition of RIPK1 kinase activity decreases serum levels of DAMPs, such as mitochondrial DNA, or markers of organ injury, such as creatinine kinase, aspartate aminotransferase, or lactate dehydrogenase $(23,48,49)$. Furthermore, DAMPs such as HMGB1 and extracellular histones, are elevated in the blood of SIRS and sepsis patients, where they have been thought to result in chronic TLR activation and tissue injury (50-52).

$\mathrm{TNF} / \mathrm{zVAD}$ administration elevated IL-1 $\beta$ levels, which were not systemically increased with TNF treatment alone. Caspase-1and caspase-1/11-deficient mice, but not IL-1 $/ 18$-deficient mice, are susceptible to TNF-induced shock, thereby excluding a primary role for caspase-1-mediated inflammasome activation in TNF shock $(23,46)$. Two recent reports have implicated MLKL activation in initiating inflammasome-mediated processing and release of IL-1 $\beta(53,54)$, suggesting that necroptosis sensitization in TNF/ zVAD-induced shock and MLKL activation may contribute to increases in plasma IL-1 $\beta$ levels.

In addition to cell death signaling, RIPK1 kinase activity has been implicated in cytokine production in vitro (32-34), including reduced transcription and production of CXCL1 in TNF/ zVAD-treated RIPK1 kinase-inactive Ripk1 $1^{\text {K5A/K45A }}$ BMDMs (22). In agreement with these recent reports, we found CXCL1, CXCL2, and IL-6 transcription and secretion impaired in vitro in TNF/zVAD-treated Ripk1 $1^{\text {D138N/D138N }}$ BMDMs (Supplemental Figure $1)$, suggesting that reductions in the cytokine response in RIPK1 
kinase-inactive mice are due to effects of RIPK1 kinase activity on cytokine production rather than necroptotic cell death. With the exception of CXCL2, however, cytokine/chemokine production at early time points is unaffected by a lack of RIPK1 kinase activity (Figures 1 and 4), demonstrating that, in vivo, RIPK1 kinase activity is not required for cytokine/chemokine induction in response to a high dose of TNF. Substantial reductions in plasma cytokine/ chemokine levels were observed in TNF-treated Ripk1 $1^{\text {D138N/D138N }}$ mice at 7 hours, consistent with the proinflammatory nature of necroptotic death. Additionally, Ripk1 $1^{D 138 N / D 138 N} \rightarrow$ WT chimeras had elevated cytokine/chemokine levels in response to TNF/zVAD administration (Supplemental Figure 3), suggesting that RIPK1 kinase activity does not regulate cytokine/chemokine expression when caspases are inhibited in vivo.

We found that 3 of 11 Ripk1 $1^{D 138 N / D 138 N} \rightarrow$ WT chimeras recovered from TNF/zVAD administration (Figure 3E), indicating that Ripk1 $1^{D 138 N / D 138 N}$ hematopoietic cells can contribute to protection. Our data suggest that optimal cytokine/chemokine induction requires necroptosis-competent (WT) hematopoietic and endothelial cells (Figure 4A), leading us to speculate that DAMPs released by necroptotic endothelial cells feed back on WT hematopoietic cells to induce necroptosis and further amplify cytokine levels. Thus, Ripk1 $1^{D 138 N / D 138 N}$ hematopoietic cells may mediate recovery in $R i p k 1^{D 138 N / D 138 N} \rightarrow$ WT chimeras by failing to undergo necroptosis in response to DAMPs released by WT necroptotic endothelial cells. Alternatively, recovery may be mediated by the survival of Ripk1 $1^{D 138 N / D 138 N}$ hematopoietic cells and production of antiinflammatory cytokines that support barrier repair.

A caveat of our bone marrow chimera studies is that protected $\mathrm{WT} \rightarrow R i p k 1^{D 138 N / D 138 N}$ mice retain radiation-resistant, tissue-resident Ripk1 $1^{D 138 N / D 138 N}$ macrophages such as Kupffer cells (KC) in the liver. $\mathrm{KC}$ associate with endothelial cells in the liver sinusoids and function to maintain liver homeostasis by surveying microbial products from the intestine. KC express TLRs and produce cytokines and chemokines in response to DAMPs or pathogen-associated molecular patterns (PAMPs). Moreover, KC undergo necroptosis upon Listeria monocytogenes infection (55). Thus, Ripk1 $1^{D 138 N / D 138 N} \mathrm{KC}$ may contribute to resistance to shock by failing to undergo necroptosis and/or produce cytokine/chemokines in response to microbial products or DAMPs released by necroptotic endothelial cells.

Although we detected decreases in cleaved caspase-3 positive intestinal epithelial cells in the ileum of TNF-treated Ripk1 $1^{D 138 N / D 138 N}$ mice, no significant differences in intestinal apoptosis were observed between TNF/zVAD-treated WT and Ripk1 ${ }^{\text {D138N/D138N }}$ mice (Supplemental Figure 4). Consistent with these data, executioner caspase-3 or caspase-7 and TNFR1-mediated intestinal epithelial cell apoptosis do not appear to contribute to TNF-induced lethality $(23,37)$. However, Newton et al. showed that, while there is evidence of slight hypothermia in Ripk3 $^{-/-}$and $\mathrm{Mlkl}^{-/}$mice in response to TNF, Ripk3 $3^{-1}$ Casp $8^{-/-}$and $\mathrm{Mlkl}^{-/-} \mathrm{Casp}^{-/-}$double-knockout mice are completely protected from TNF-induced hypothermia and have significantly reduced serum cytokine and chemokine levels (47). Additionally, they showed that $C a s p 8^{+/-}$and Villin-cre Ripk $3^{f / f l}$ mice are largely protected from TNF-induced cytokine/chemokine expression and hypothermia, suggesting that caspase- 8 contributes to lethality during TNF-induced shock (47). Since our data and others indicate no role for the caspase-1-mediated inflammasome or intestinal apoptosis in TNF-induced lethality $(23,37,46)$, caspase-8-mediated effects likely reflect its role or roles in inflammasome-independent IL-1 $\beta$ regulation and processing (56).

Although TNF shock is typically thought of as a sterile model of injury, several reports show a key role for intestinal barrier dysfunction and systemic spread of bacteria in the blood of TNF-treated mice $(36,37)$. While intestinal necroptosis was not addressed in the Newton study, the Villin-cre Ripk $3^{\text {fl/fl }}$ mice may be protected from hypothermia and increased cytokine levels because RIPK3-deficient intestinal epithelial cells fail to undergo necroptosis, and consequently, the intestinal epithelial barrier is maintained. We show that Ripk1 ${ }^{D 138 N / D 138 N}$ mice did not exhibit TNF-induced increases in intestinal permeability, and we did not detect spread of bacteria to peripheral organs, such as the liver or spleen. While breaches in the intestinal epithelium and entry of bacteria or bacterial products into the bloodstream may contribute to TNF-induced shock, our data demonstrate that the downstream effects of the host response converge on the vascular endothelium.

We found that TNF-resistant Ripk1 $1^{\text {D138N/D138N }}$ mice and WT $\rightarrow$ Ripk1 $1^{D 138 N / D 138 N}$ chimeras did not exhibit increases in lung and liver vascular permeability or evidence of increased tissue factor expression and coagulation. Furthermore, in the TNF/zVAD model, vascular barrier protection and an absence of coagulation, at least in the liver, correlated with an inability to detect phospho-MLKL reactivity in the RIPK1 kinase-inactive endothelium. Our results indicate that necroptosis contributes to barrier disruption and activation of the clotting cascade, as we found MLKL activation in liver endothelial cells isolated from TNF/zVAD-treated WT mice.

Although TNF and TNF/zVAD treatment increased plasma tissue factor levels (Figure 5D) and induced coagulation (Figure 6, A and B), we detected fewer breaks in the liver endothelium of TNF-treated WT mice (Figure 6D). Consistent with this observation, we were unable to detect phospho-MLKL-reactive endothelial cells in livers of TNF-treated mice (Supplemental Figure 8A). We hypothesized that TNF may stimulate RIPK1 kinase-dependent apoptosis; however, cleaved caspase-3-reactive cells were not detected in TNF-treated liver sections by immunohistochemistry or by immunoblotting endothelial cell lysates from TNF-treated mice (Supplemental Figure 8, B and C), suggesting that apoptosis is not responsible for the vascular breaks. In contrast with TNF/ zVAD administration, in which hypothermia and lethality are rapid and fully penetrant in WT controls (ref. 21 and Figure 3), mice treated with TNF took longer to exhibit symptoms (hypothermia, inactivity, hunched posture) and were variable in their presentation at sacrifice, 8 hours after TNF administration. We speculate that our inability to detect phospho-MLKL-reactive endothelial cells in mice treated with TNF reflects this variability in presentation, and consequently, fewer endothelial breaks (Figure 6D) and potentially less endothelial cell necroptosis were induced following TNF treatment for 8 hours compared with TNF/zVAD treatment for 4 hours.

We also demonstrate that lung endothelial cells isolated from WT, but not Ripk1 $1^{D 138 N / D 138 N}$, mice undergo necroptosis in vitro when treated with TNF, Smac, and zVAD, but not with TNF/zVAD only (Supplemental Figure 5C). This finding was unexpected, as TNF/ zVAD administration is sufficient to induce liver endothelial cell necroptosis in vivo (Figure 6E). The requirement for Smac mimet- 
ic to induce endothelial necroptosis in vitro may reflect increased cellular pools of cIAP1/2 or XIAP in lung versus liver endothelial cells or simply indicate that TNF/zVAD concentrations achieved in vivo are not accurately modeled in vitro.

Many septic patients have impaired endothelial barrier function and exhibit edema, coagulation abnormalities, and decreased levels or dysfunctional activation of anticoagulants. The most promising clinical trials have focused on combining antiinflammatory and anticoagulant agents, such as activated protein $\mathrm{C}$ or thrombomodulin (57). Recent advances focus on methods to strengthen the vascular endothelium and modulate its response to proinflammatory cytokine levels. In several models of LPS endotoxemia or cecal ligation and puncture, enhanced vascular stability dramatically increased mouse survival, even though no reductions in the proinflammatory cytokine response were observed $(58,59)$. Similarly, we demonstrate that chimeric mice susceptible to TNF-induced shock exhibited increased vascular permeability and coagulation even when proinflammatory cytokine levels were not sustained (Figure 4A and Figure 5, E and F). We provide evidence that Ripk1 $1^{D 138 N / D 138 N}$ liver endothelial cells failed to exhibit MLKL activation and undergo necroptosis (Figure 6E), thereby maintaining an intact vasculature that protects RIPK1 kinaseinactive mice from TNF/zVAD-induced lethality. Interestingly, RIPK3 plasma levels are significantly increased in nonsurvivors of sepsis (60), suggesting that endothelial cell necroptosis may contribute to lethality in both SIRS and sepsis patients. Ripk1 $1^{D 138 N / D 138 N}$, $\mathrm{Ripk3}^{-/-}$, and $\mathrm{Mlkl}^{-/-}$mice have been examined in mouse models of sepsis, and although the results generated using the cecal ligation and puncture model are conflicting $(15,23,61)$, we and others find Ripk1 $1^{D 138 N / D 138 N}$ mice susceptible to LPS administration (ref. 47 and M. Zelic and M.A. Kelliher, unpublished observations), indicating that necroptosis may not be a critical factor in sepsis. The role of necroptosis in shock and sepsis needs to be explored further, but our results demonstrate that RIPK1 kinase inhibitors may show promise in ameliorating or preventing inflammatory vascular injury and SIRS.

\section{Methods}

Mice. Ripk1 $1^{D 138 N / D 138 N}$ mice on a C57BL/6 background were described previously (21). For bone marrow transplantation studies, recipient

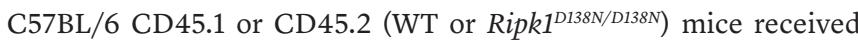
$11 \mathrm{~Gy}$ of total body irradiation in a split dose (5.5 Gy/dose) using a Cesium-137 irradiator, with a 4 -hour rest between doses. Irradiated recipients were reconstituted by i.v. injection of $2 \times 10^{6} \mathrm{WT}$ (CD45.1 or CD45.2) or Ripk1 $1^{D 138 N / D 138 N}$ (CD45.2) bone marrow cells. Recipients were maintained on medicated water for 1 month. Cheek bleeds were performed after 4 and 8 weeks to assess reconstitution efficiency by flow cytometry.

TNF-induced shock experiments. Sex-matched male WT and Ripk1 ${ }^{D 138 N / D 138 N}$ littermates were injected i.v. with $9 \mu \mathrm{g}$ mTNF- $\alpha$ (Cell Sciences) alone or in combination with $16.7 \mathrm{mg} / \mathrm{kg}$ zVAD-fmk (Bachem) unless noted otherwise. Body temperature and survival were monitored every few hours. Mice were used between 8 and 12 weeks of age. For ex vivo analyses, mice were typically sacrificed after 4 (TNF/zVAD) or 8 hours (TNF). Reconstituted mice were injected with mTNF- $\alpha$ and zVAD-fmk 2 months after reconstitution. When littermate controls were not used, sex- and age-matched mice were cohoused.
Cytokine analysis. Plasma cytokines were measured using a 12-plex protein/peptide multiplex analysis (Luminex Technology) conducted by the National Mouse Metabolic Phenotyping Center at the University of Massachusetts Medical School. Cytokines and chemokines that were below the limit of detection were assigned a value of zero. Blood was collected via cardiac puncture in a heparinized needle and spun down to isolate plasma. The samples were stored at $-80^{\circ} \mathrm{C}$ until analysis. Plasma tissue factor levels were determined by ELISA (R\&D Systems). IL-6 (eBioscience), CXCL1, CXCL2, and CCL5 (R\&D Systems) secretion by cultured primary cells was determined by ELISA.

Gene-expression analysis. Primary BMDMs were treated with 50 $\mathrm{ng} / \mathrm{ml} \mathrm{TNF}$ (R\&D Systems) or TNF and $50 \mu \mathrm{M} z \mathrm{zAD}$ (Enzo) for 2 or 6 hours. Primary lung endothelial cells were treated with TNF (100 ng/ $\mathrm{ml}$ ) for 1 or 4 hours. Total cellular RNA was isolated using an RNeasy Mini Kit (QIAGEN). Total lung or liver RNA was extracted with TRIzol (Life Technologies). cDNA was prepared using the SuperScript FirstStrand Synthesis System (Invitrogen). Quantitative real-time PCR was performed using Power SYBR Green PCR Master Mix (Applied Biosystems) and run on the AB7300 Detection System (Applied Biosystems). Relative gene expression was normalized to $\beta$-actin and determined using the $\Delta \Delta \mathrm{CT}$ method. The primer sequences used to amplify murine genes are listed in Supplemental Table 1.

Flow cytometry. Single-cell suspensions from blood, spleen, bone marrow, lung, and liver were stained with cell-surface antibodies for myeloid (Gr1, CD11b, CD11c, and Ly6c), lymphoid (CD4, CD8, and B220), and endothelial (CD31) markers. To distinguish between donor and host hematopoietic cells in the bone marrow reconstitution experiments, anti-CD45.1 and anti-CD45.2 antibodies were used. DAPI (Molecular Probes) was used to distinguish between live and dead cells. All samples were run on a BD LSRII flow cytometer (BD Bioscience) and analyzed using FlowJo software (Tree Star). A complete list of flow cytometry antibodies is provided in Supplemental Table 2.

Prothrombin time test. Blood was collected via cardiac puncture and mixed with $3.2 \%$ sodium citrate at a 9:1 ratio. Plasma was isolated and the test was conducted by IDEXX.

Vascular permeability assay. A 1\% Evans blue (Sigma-Aldrich) solution was injected i.v. at $40 \mathrm{mg} / \mathrm{kg} 6$ hours after $\mathrm{mTNF}-\alpha$ administration. After 1 hour, the mice were sacrificed and perfused, and organs were dried overnight before homogenization in formamide (MilliporeSigma). The amount of extracted Evans blue dye was assessed by spectrophotometry at $620 \mathrm{~nm}$ on a Beckman Coulter DTX880 plate reader.

Intestinal permeability assay. Mice were orally gavaged with 100 $\mu \mathrm{l}$ of $150 \mathrm{mg} / \mathrm{kg}$ FITC-dextran (M.W. 4000) beads (Sigma-Aldrich) 2 hours after TNF administration. Six hours later, blood was drawn via cardiac puncture. The amount of FITC-dextran in plasma was measured on a Beckman Coulter DTX880 plate reader (excitation wavelength, $488 \mathrm{~nm}$; emission wavelength, $530 \mathrm{~nm}$ ).

Histology and immunohistochemistry. Livers were fixed in 2\% paraformaldehyde (Fisher Scientific). Samples were embedded in paraffin, and H\&E staining was done on $8 \mu \mathrm{m}$-thick sections according to standard protocols. Cleaved caspase-3 staining was done on $6 \mu \mathrm{m}-$ thick sections at 1:100 dilution of cleaved caspase-3 antibody (catalog 9661, Cell Signaling Technology) according to standard protocols. For endomucin staining, $3 \mu \mathrm{m}$-thick liver sections were processed through xylene and rehydrated through an ethanol gradient prior to immersion in sodium citrate buffer (10 mM sodium citrate, $0.05 \%$ Tween $20, \mathrm{pH}$ 6) and antigen retrieval was done using a 2100 Antigen Retriever. Sec- 
tions were incubated for 1 hour at room temperature (RT) in blocking solution (1\% rabbit serum, 1\% BSA, 0.3\% Triton-X 100 in PBS) and then kept in a primary antibody mix (Endomucin, 1:200, Santa Cruz Biotechnology Inc., sc-65495; in 2.5\% rabbit serum in PBS) overnight at $4^{\circ} \mathrm{C}$. Rat secondary antibody was used for 1 hour at RT (Vector Biotinylated Anti-Rat IgG). Sections were developed using the Vectastain Elite ABC Kit and the DAB Peroxidase Substrate Kit according to the manufacturer's instructions (Vector Laboratories) before counterstaining with hematoxylin and mounting with VECTASHIELD Dry Mounting Media. All images were acquired using a Nikon Eclipse 80i microscope. To quantify the percentage of clotting in liver blood vessels, 8 random fields at a $\times 20$ field of view were counted per mouse. To quantify the number of cleaved caspase-3-positive cells in the intestines or liver, 10 random fields at $\times 40$ field of view were counted per mouse.

Primary cell cultures. BMDMs were generated by culturing bone marrow cell suspensions in $20 \%$ conditioned media from L929 cells for 7 days. On day 7, adherent cells were replated in 6-well plates for mRNA experiments $\left(2 \times 10^{6}\right.$ cells per well $)$ or in 96-well plates for ELISA assays $\left(2 \times 10^{5}\right.$ cells per well). Mouse lungs were digested in 25 $\mathrm{ml}$ of $1 \mathrm{mg} / \mathrm{ml}$ collagenase at $37^{\circ} \mathrm{C}$ for an hour. After sequential filtering through a $100 \mu \mathrm{m}$ and $40 \mu \mathrm{m}$ cell strainer, the cell suspension was plated on a gelatin-coated T75 flask and the cells were incubated for 4 to 6 days until reaching confluence. Cells were grown in endothelial cell media containing low-glucose DMEM/F-12 with 20\% FBS, $1 \%$ L-glutamine, $1 \%$ penicillin/streptomycin, $100 \mu \mathrm{g} / \mathrm{ml}$ heparin, and $50 \mu \mathrm{g} / \mathrm{ml}$ endothelial cell growth supplement (Biomedical Technologies Inc.). To immunoselect endothelial cells, confluent T75 flasks were incubated with CD-31 (PECAM-1, catalog 553369, BD Biosciences) and CD102-coated (ICAM-2, catalog 553325, BD Biosciences) Dynabeads on ice for 20 minutes. Using a magnetic separator, beadbound cells were recovered and washed 3 times with PBS. Endothelial cells were plated on gelatin-coated T25 flasks and expanded.

Liver endothelial cell isolation. Mice were perfused with PBS, and livers were minced with a razor blade on ice and shaken for 30 minutes at $37^{\circ} \mathrm{C}$ in $1 \mathrm{mg} / \mathrm{ml}$ collagenase. The suspension was filtered through a $100 \mu \mathrm{m}$ strainer and spun for 5 minutes at $50 \mathrm{~g}$ to separate out hepatocytes. The supernatant was spun again for 5 minutes at $60 \mathrm{~g}$ before the nonparenchymal cell fraction in the supernatant was pelleted at 700 $g$ for 10 minutes. After red blood cell lysis, the nonparenchymal liver cell fraction was depleted of $\mathrm{CD} 45^{+}$cells before enriching for $\mathrm{CD} 146^{+}$ endothelial cells with MACS MicroBeads, according to the manufacturer's instructions (Miltenyi Biotec). Purity was checked by FACS, and recovered cells were snap-frozen to use for Western blot.
Immunoblotting. Isolated liver endothelial cells were lysed in RIPA buffer (150 mM NaCl, $50 \mathrm{mM}$ Tris- $\mathrm{HCl}$ [pH 7.5], 1\% NP40, 0.25\% deoxycholate, $0.1 \%$ SDS, $1 \mathrm{mM}$ EDTA) supplemented with protease inhibitors (Roche Applied Science), sodium orthovanadate (1 mM), PMSF (1 mM), and DTT (1 mM). Total protein was run on a $10 \%$ SDSPAGE gel, transferred to a nitrocellulose membrane (Invitrogen), and probed with antibodies to phospho-MLKL (ab196436, Abcam), MLKL (gift from Jiahuai Han, Xiamen University, Xiamen, China, and AP14272b, Abgent), caspase-3 (catalog 9662, Cell Signaling), and Erk1/2 (catalog 9102, Cell Signaling). Membranes were developed with Clarity Western ECL Substrate (Bio-Rad).

Cell viability. For apoptosis and necroptosis assays, cultured lung endothelial cells were pretreated with $1 \mu \mathrm{M} \mathrm{Smac}$ mimetic (ChemieTek) or $20 \mu \mathrm{M}$ zVAD-fmk (Enzo) prior to adding TNF (10 ng/ml). Metabolic activity was assayed after 20 hours by the addition of MTS reagent (CellTiter 96 AQueous One Solution Cell Proliferation Assay, Promega) and measured using a Beckman Coulter DTX880 plate reader. Absorbance values at $535 \mathrm{~nm}$ were normalized to Smac mimetic control.

Statistics. Data are presented as mean \pm SEM. Statistical analyses were performed using GraphPad Prism software, version 7.02. Kaplan-Meier survival curves were analyzed using a log-rank test with a $95 \%$ CI. Statistical analysis was performed using the unpaired 2-tailed Student's $t$ test or 1-way ANOVA or 2-way ANOVA with post hoc Tukey's test.

Study approval. All animal procedures used in this study were approved by the University of Massachusetts Medical School IACUC and conducted in accordance with university guidelines.

\section{Author contributions}

MZ, JER, and MAK designed research. MZ, JER, JAO, JL, SEL, and HPJ performed experiments. MZ, JER, JAO, and CMT analyzed experiments. CMT and MP provided reagents. MZ and JL prepared figures. MZ and MAK wrote the paper.

\section{Acknowledgments}

This work was supported by NIH grants AI075118 (to MAK) and HL118100 (to CMT). MZ was supported by Innate Immunity T32 Training Grant AI095213.

Address correspondence to: Michelle A. Kelliher, Department of Molecular, Cell and Cancer Biology, Lazare Research Building, 364 Plantation Street, Worcester, Massachusetts 01605, USA. Phone: 508.856.8620; Email: michelle.kelliher@umassmed.edu.
1. Pasparakis M, Vandenabeele P. Necroptosis and its role in inflammation. Nature. 2015;517(7534):311-320.

2. Weinlich R, Oberst A, Beere HM, Green DR. Necroptosis in development, inflammation and disease. Nat Rev Mol Cell Biol. 2016;18(2):127-136.

3. Kaczmarek A, Vandenabeele P, Krysko DV. Necroptosis: the release of damage-associated molecular patterns and its physiological relevance. Immunity. 2013;38(2):209-223.

4. Kelliher MA, Grimm S, Ishida Y, Kuo F, Stanger BZ, Leder P. The death domain kinase RIP mediates the TNF-induced NF-kappaB signal. Immunity. 1998;8(3):297-303.
5. Dillon CP, et al. RIPK1 blocks early postnatal lethality mediated by caspase- 8 and RIPK3. Cell. 2014;157(5):1189-1202.

6. Rickard JA, et al. RIPK1 regulates RIPK3-MLKLdriven systemic inflammation and emergency hematopoiesis. Cell. 2014;157(5):1175-1188.

7. Kaiser WJ, et al. RIP1 suppresses innate immune necrotic as well as apoptotic cell death during mammalian parturition. Proc Natl Acad Sci U S A. 2014;111(21):7753-7758.

8. Dannappel M, et al. RIPK1 maintains epithelial homeostasis by inhibiting apoptosis and necroptosis. Nature. 2014;513(7516):90-94.

9. Takahashi N, et al. RIPK1 ensures intestinal homeostasis by protecting the epithelium against apoptosis. Nature. 2014;513(7516):95-99.

10. Roderick JE, et al. Hematopoietic RIPK1 deficiency results in bone marrow failure caused by apoptosis and RIPK3-mediated necroptosis. Proc Natl Acad Sci U S A. 2014;111(40):14436-14441.

11. He S, Liang Y, Shao F, Wang X. Toll-like receptors activate programmed necrosis in macrophages through a receptor-interacting kinase-3mediated pathway. Proc Natl Acad Sci US A. 2011;108(50):20054-20059.

12. Kaiser WJ, Sridharan H, Huang C, et al. Toll-like receptor 3-mediated necrosis via TRIF, RIP3, and MLKL. J Biol Chem. 2013;288(43):31268-31279. 
13. Upton JW, Kaiser WJ, Mocarski ES. DAI/ZBP1/ DLM-1 complexes with RIP3 to mediate virusinduced programmed necrosis that is targeted by murine cytomegalovirus vIRA. Cell Host Microbe. 2012;11(3):290-297.

14. Newton K, Sun X, Dixit VM. Kinase RIP3 is dispensable for normal NF-kappa Bs, signaling by the B-cell and T-cell receptors, tumor necrosis factor receptor 1, and Toll-like receptors 2 and 4. Mol Cell Biol. 2004;24(4):1464-1469.

15. Wu J, et al. Mlkl knockout mice demonstrate the indispensable role of Mlkl in necroptosis. Cell Res. 2013;23(8):994-1006.

16. Murphy JM, et al. The pseudokinase MLKL mediates necroptosis via a molecular switch mechanism. Immunity. 2013;39(3):443-453.

17. Degterev A, et al. Chemical inhibitor of nonapoptotic cell death with therapeutic potential for ischemic brain injury. Nat Chem Biol. 2005;1(2):112-119.

18. Degterev A, et al. Identification of RIP1 kinase as a specific cellular target of necrostatins. Nat Chem Biol. 2008;4(5):313-321.

19. Takahashi N, et al. Necrostatin-1 analogues: critical issues on the specificity, activity and in vivo use in experimental disease models. Cell Death Dis. 2012;3(11):e437.

20. Newton K, et al. Activity of protein kinase RIPK3 determines whether cells die by necroptosis or apoptosis. Science. 2014;343(6177):1357-1360.

21. Polykratis A, et al. Cutting edge: RIPK1 kinase inactive mice are viable and protected from TNF-induced necroptosis in vivo. J Immunol. 2014;193(4):1539-1543.

22. Berger SB, et al. Cutting Edge: RIP1 kinase activity is dispensable for normal development but is a key regulator of inflammation in SHARPIN-deficient mice. JImmunol. 2014;192(12):5476-5480.

23. Duprez L, et al. RIP kinase-dependent necrosis drives lethal systemic inflammatory response syndrome. Immunity. 2011;35(6):908-918.

24. Matsuda N, Hattori Y. Systemic inflammatory response syndrome (SIRS): molecular pathophysiology and gene therapy. JPharmacol Sci. 2006;101(3):189-198.

25. Singer M, et al. The Third International Consensus Definitions for Sepsis and Septic Shock (Sepsis-3). JAMA. 2016;315(8):801.

26. Opal SM, et al. Confirmatory interleukin-1 receptor antagonist trial in severe sepsis: a phase III, randomized, double-blind, placebo-controlled, multicenter trial. The Interleukin-1 Receptor Antagonist Sepsis Investigator Group. Crit Care Med.1997;25(7):1115-1124

27. Abraham E, et al. Lenercept (p55 tumor necrosis factor receptor fusion protein) in severe sepsis and early septic shock: a randomized, double-blind, placebo-controlled, multicenter phase III trial with 1,342 patients. Crit Care Med. 2001;29(3):503-510.

28. Abraham E, et al. Double-blind randomised controlled trial of monoclonal antibody to human tumour necrosis factor in treatment of septic shock. NORASEPT II Study Group. Lancet.
1998;351(9107):929-933.

29. Cauwels A, Janssen B, Waeytens A, Cuvelier C, Brouckaert P. Caspase inhibition causes hyperacute tumor necrosis factor-induced shock via oxidative stress and phospholipase A2. Nat Immunol. 2003;4(4):387-393.

30. Machado JR, et al. Neonatal sepsis and inflammatory mediators. Mediators Inflamm. 2014;2014:269681.

31. Sjövall F, Morota S, Frostner EÅ, Hansson MJ, Elmér E. Correction: Cytokine and nitric oxide levels in patients with sepsis - temporal evolvement and relation to platelet mitochondrial respiratory function. PLoS One. 2014;9(7):e103756

32. Najjar M, et al. RIPK1 and RIPK3 kinases promote cell-death-independent inflammation by Toll-like receptor 4. Immunity. 2016;45(1):46-59.

33. Shutinoski B, et al. K45A mutation of RIPK1 results in poor necroptosis and cytokine signaling in macrophages, which impacts inflammatory responses in vivo. Cell Death Differ. 2016;23(10):1628-1637.

34. Christofferson DE, et al. A novel role for RIP1 kinase in mediating TNF $\alpha$ production. Cell Death Dis. 2012;3(6):e320.

35. Kruger P, et al. Neutrophils: Between host defence, immune modulation, and tissue injury. PLoS Pathog. 2015;11(3):e1004651.

36. Van Hauwermeiren F, et al. Safe TNF-based antitumor therapy following p55TNFR reduction in intestinal epithelium. J Clin Invest. 2013;123(6):2590-2603.

37. Van Hauwermeiren F, et al. TNFR1-induced lethal inflammation is mediated by goblet and Paneth cell dysfunction. Mucosal Immunol. 2015;8(4):828-840.

38. Aird WC. The role of the endothelium in severe sepsis and multiple organ dysfunction syndrome. Blood. 2003;101(10):3765-3777.

39. Mai J, Virtue A, Shen J, Wang H, Yang X-F. An evolving new paradigm: endothelial cells--conditional innate immune cells. J Hematol Oncol. 2013;6(1):61.

40. Iyoda M, et al. IL-17A and IL-17F stimulate chemokines via MAPK pathways (ERK1/2 and p38 but not JNK) in mouse cultured mesangial cells: synergy with TNF-alpha and IL-1beta. $A m J$ Physiol Renal Physiol. 2010;298(3):F779-F787.

41. Brackett CM, Muhitch JB, Evans SS, Gollnick SO. IL-17 promotes neutrophil entry into tumor-draining lymph nodes following induction of sterile inflammation. J Immunol. 2013;191(8):4348-4357.

42. Sparna T, et al. Genome-wide comparison between IL-17 and combined TNF-alpha/IL-17 induced genes in primary murine hepatocytes. BMC Genomics. 2010;11(1):226.

43. He S, et al. Receptor interacting protein kinase-3 determines cellular necrotic response to TNF- $\alpha$. Cell. 2009;137(6):1100-1111.

44. Morgan SM, Samulowitz U, Darley L, Simmons DL, Vestweber D. Biochemical characterization and molecular cloning of a novel endothelialspecific sialomucin. Blood. 1999;93(1):165-175 45. Takahashi N, et al. IL-17 produced by Paneth cells drives TNF-induced shock. JExp Med. 2008;205(8):1755-1761.

46. Berghe T Vanden, et al. Simultaneous targeting of IL-1 and IL-18 is required for protection against inflammatory and septic shock. Am J Respir Crit Care Med. 2014;189(3):282-291.

47. Newton K, et al. RIPK3 deficiency or catalytically inactive RIPK1 provides greater benefit than MLKL deficiency in mouse models of inflammation and tissue injury. Cell Death Differ. 2016;23(9):1565-1576.

48. Najjar M, et al. Structure guided design of potent and selective ponatinib-based hybrid inhibitors for RIPK1. Cell Rep. 2015;10(11):1850-1860.

49. Ren Y, et al. Discovery of a highly potent, selective, and metabolically stable inhibitor of receptor-interacting protein 1 (RIP1) for the treatment of systemic inflammatory response syndrome. JMed Chem. 2017;60(3):972-986.

50. Kutcher ME, Xu J, Vilardi RF, Ho C, Esmon CT, Cohen MJ. Extracellular histone release in response to traumatic injury. J Trauma Acute Care Surg. 2012;73(6):1389-1394.

51. Lotze MT, Tracey KJ. High-mobility group box 1 protein (HMGB1): nuclear weapon in the immune arsenal. Nat Rev Immunol. 2005;5(4):331-342.

52. Xu J, et al. Extracellular histones are major mediators of death in sepsis. Nat Med. 2009;15(11):1318-1321.

53. Gutierrez KD, et al. MLKL activation triggers NLRP3-mediated processing and release of IL-1 1 independently of gasdermin-D. J Immunol. 2017;198(5):2156-2164.

54. Conos SA, et al. Active MLKL triggers the NLRP3 inflammasome in a cell-intrinsic manner. Proc Natl Acad Sci U S A. 2017;114(6):E961-E969.

55. Blériot C, Dupuis T, Jouvion G, Eberl G, Disson O, Lecuit M. Liver-resident macrophage necroptosis orchestrates type 1 microbicidal inflammation and type-2-mediated tissue repair during bacterial infection. Immunity. 2015;42(1):145-158.

56. Gurung P, Kanneganti T-D. Novel roles for caspase-8 in IL-1b and inflammasome regulation. Am J Pathol. 2015;185(1):17-25.

57. Levi M, van der Poll T. Coagulation and sepsis. Thromb Res. 2017;149:38-44.

58. London NR, et al. Targeting Robo4-dependent Slit signaling to survive the cytokine storm in sepsis and influenza. Sci Transl Med. 2010;2(23):23ra19.

59. Xu H, Ye X, Steinberg H, Liu SF. Selective blockade of endothelial NF-kB pathway differentially affects systemic inflammation and multiple organ dysfunction and injury in septic mice. JPathol. 2009;220(4):490-498.

60. Qing DY, et al. Red blood cells induce necroptosis of lung endothelial cells and increase susceptibility to lung inflammation. Am J Respir Crit Care Med. 2014;190(11):1243-1254.

61. Sharma A, Matsuo S, Yang W-L, Wang Z, Wang P. Receptor-interacting protein kinase 3 deficiency inhibits immune cell infiltration and attenuates organ injury in sepsis. Crit Care. 2014;18(4):R142. 